\title{
Integration Technology of Multi-platform Models Supporting Visual Simulation
}

\author{
Yanyan Huang *, Hanghai Wu \\ School of automation, Nanjing University of Science \& Technology, Nanjing 210094, China \\ * Corresponding author. email: imhyy@sina.com \\ Manuscript submitted June 10, 2016; accepted August 20, 2016. \\ doi: 10.17706/jsw.11.11.1162-1171
}

\begin{abstract}
It is difficult to integrate different 3D models to support the simulation application in Vega Prime tool, and thus, we research the simplification and integration methods for the popular 3D modeling tools(3ds Max, Maya, etc.) and extremely rich models developed by those tools to the simulation development. This paper puts forward a complete set of methods for model format conversion and model structure optimization in order to make these models suitable for simulation development. By Importing the popular 3D modeling tools, we can solves the problem which developed for building models for Vega Prime only depend on Creator and is not friendly enough to users; importing those existed 3D models developed by the popular modeling tools enriches the model base of simulation, greatly reduces the work of simulation development and shortens the time of simulation development. This method which is based on format conversion and takes some model simplification methods to integrate models from different platforms into a unified Vega Prime simulation platform greatly enhances the ability of Vega Prime simulation development. That also provides a new way for those visual simulation developers who own poor ability of modeling.
\end{abstract}

Key words: 3D model, simulation, multi-platform modeling, format conversion, model optimization.

\section{Introduction}

Vega Prime is software which is introduced by MultiGen-Paradigm Company for the field of real-time visual simulation, sound simulation and virtual reality [1]. Vega Prime is a real time visual simulation platform, it has a high demand for the visual effect and real-time performance of the simulation. Visual effect needs simulation scene owning lots of details to fit the actual scene, and real-time requirements needs the refresh rate of pictures in scene simulation reach at least 15 frames per second or more, and can respond to the interactive control in time. To achieve the visual effect, the model needs to be refined and detailed, which makes the model more complicated. A huge amount of model data is unfavorable for real-time rendering software like Vega obviously. In order to achieve a balance between the visual effect and real time, MultiGen-Paradigm Company developed Open Flight (flt) 3D model database which has logical and hierarchical characteristics, it also developed by Creator which is a specialized modeling tool for Open Flight format 3D modeling[2]. Through the combination of Creator, Vega Prime and other Vega Prime plug-ins, the simulation can be quickly and well developed.

In the simulation development process, while the content of simulation application has been determined, the first step is to build models. Creator supports two ways of modeling. The first way is by using creator 
based geometric components and transform operations; the second way is to import existed models and then modify those models. The first way needs the operator owns high modeling abilities. If the operator is not good enough at modeling, he can hardly build a good looking model; and the time of development will be long. That reduces the efficiency of simulation development. Especially when facing unconventional emergencies, simulation is difficult to put into practice in time[3]. The second way needs a lot of existed models, although it can greatly accelerate development rate, but considered that Creator only support these types of FLT, STL, VSB, obj, 3DS, so it cannot provide enough suitable models. Although there are so many different 3D computer model formats, all formats follow the same description of 3D model standard, and then you can convert other three-dimensional model formats to FLT 3D model format. The efficiency of simulation application development can be effectively improved by using the extended second modes.

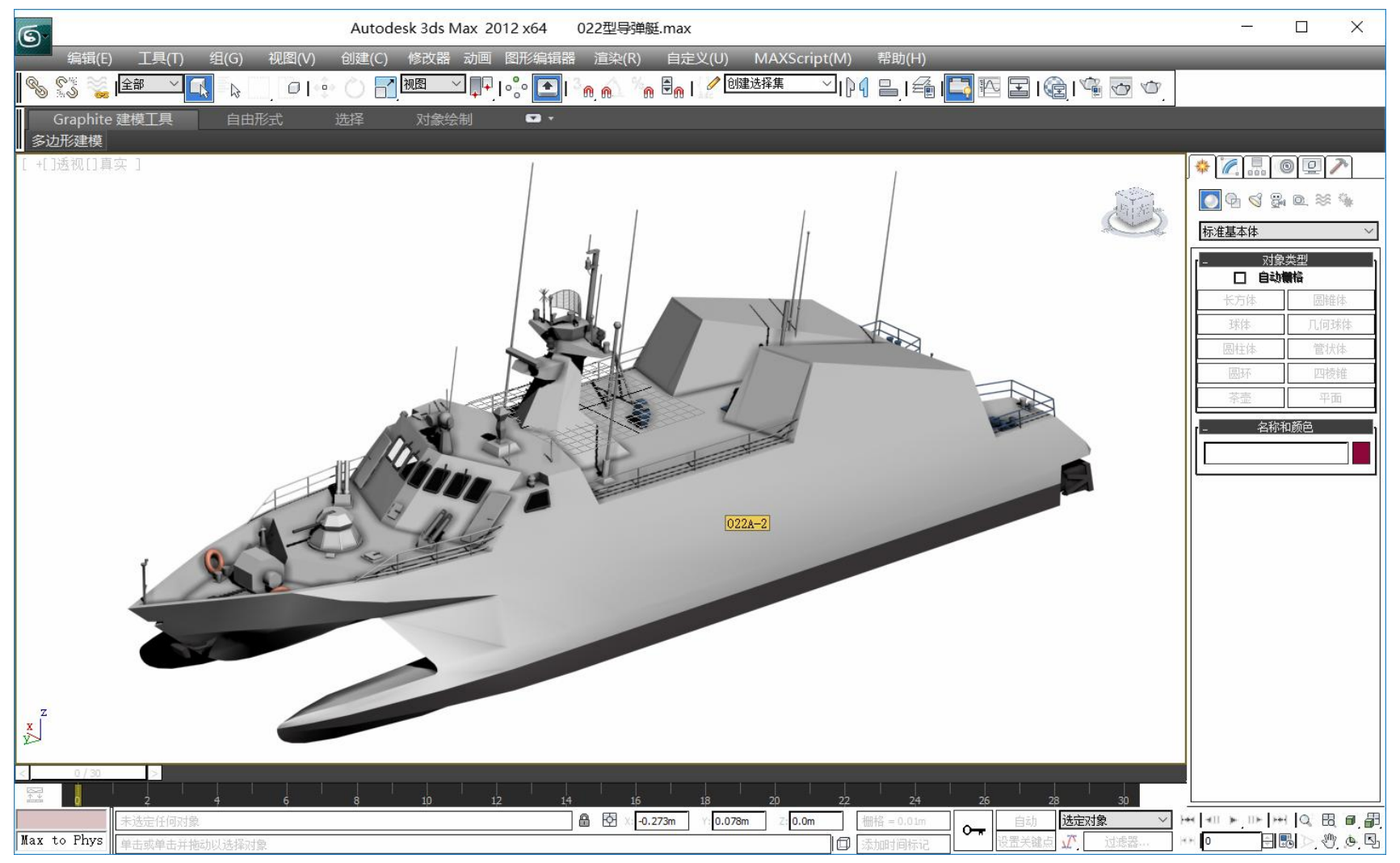

Fig. 1. Missile craft in 3ds max.

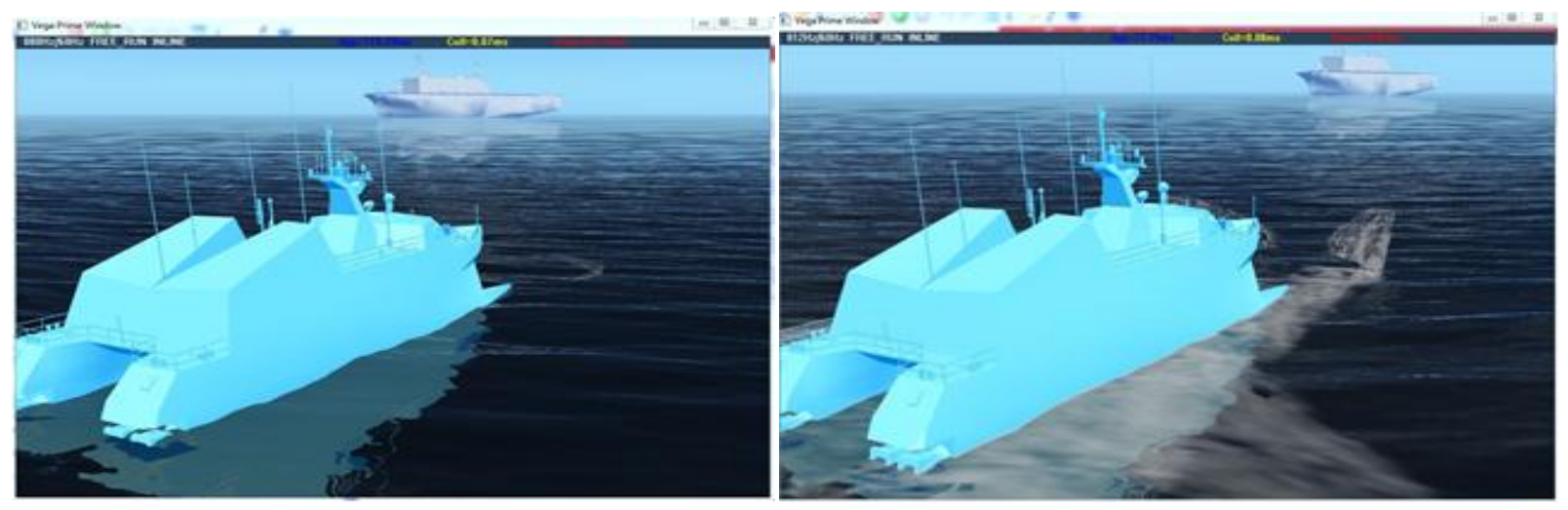

Fig. 2. Results without optimization Fps=8 (static). Fig. 3. Results with optimization Fps=12 (moving).

By using methods provided by this paper, a simulation is built much easier and faster than before. Take a 
process of developing simulation as an example, it has found that those methods are very effective. In this simulation, certain type missile craft and aircraft carrier groups are involved. By using those methods provided in this paper, the whole simulation just cost one and a half day while it may cost a week without those methods. The simulation simulates the performance of that missile craft in the offshore defense [4]. The missile craft is shown in Fig. 1. Its origin format is max. By converting the file into $3 \mathrm{ds}$ by $3 \mathrm{~d}$ s Max and then into flt by self-writing program, this model can be used in Vega Prime directly while it fps cannot reach the in-time standard. As shown in Fig. 2, the Fps is just 8 while the whole scene is static. To get a better performance, this paper provides ways of optimizing models. After optimization, the simulation can run smoothly and that is shown in Fig. 3. The fps is 12 while all models are moving in the scene.

\section{Mainstream 3d Model Format and OpenFlight}

Along with the popularity of 3D animation and 3D games, there are a lot of 3D models available on the Internet. These models' formats are different, but mostly are developed by the mainstream modeling tool.

The current mainstream models can be classified as the professional model and CG model. The professional models are models such as CAD model, and the CG models are models built by Max, 3DS, Maya and even the game engine. The main file formats are C4d, Max, 3ds, FBX, obj, DWG, DXF, ma/mb, SKP, sldprt, STL, poser/daz, blend, and etc. Each format has its corresponding modeling tools and field.

In this paper, models mainly are developed by $3 \mathrm{~d}$ s Max and Maya. It is worth mentioned, $3 \mathrm{~d}$ s Max began to support exporting OpenFlight format since version 2010. That means we can use 3ds Max directly to develop OpenFlight format models, and compared with Creator, $3 \mathrm{ds}$ Max is more humane.

\subsection{Introduction of $3 \mathrm{ds}$ Max and $3 \mathrm{ds}$ Format}

3D Max Studio, often referred to as 3ds Max or 3d Max, is a three-dimensional animation rendering and production software based on PC system. It is developed by Discreet which is a subsidiary of Autodesk Company. 3dS Max is cost-effective and also has low requirements of the hardware system. 3ds Max is friendlier to beginners, its production process is very simple and efficient [5]. Because its features of cost-effective and it is easy to use, it has a wide range of user base. 3ds Max has become a mainstream modeling tool, and there are a large number of $3 \mathrm{~d}$ s Max models on the Internet uploaded by those users .Figure 1 also shows the interface of $3 \mathrm{ds}$ Max.

3ds Max original file format is 3DS format, with the change of the version, the current default storage format is max format. The current max file is a compound document structure; the file structure is not open. Max file uses the plug-ins, each plug-in has its own storage structure. Max plug-in source is various, so the max file storage structure is difficult to learn. We can achieve the max data by learning the content of the max SDK. 3DS file format is also a classic 3D model format. Writing format conversion program must use the interpretation of its file structure [6].

3DS file is made up of "chunk". They describe the data that is connected to them. The "chunk" is composed of two parts: 1.ID; 2.The location of the next data block.Each chunk is a hierarchy that is represented by the ID. 3ds has a main chunk, its ID is 0x4D4D .The main chunk is 3DS file start, then there are various sub chunks, such as edit sub chunk, object sub chunk, camera sub chunk, light source sub chunk, triangular polygon list sub chunk, key frame sub chunk, color chunk, and view-port chunk [7]. 3D edit chunk is a chunk that describes the data of the 3D object. 3D object is stored in this chunk. The key frame chunk describes the key frame data.

\section{MAIN3DS (0x4D4D) //Main chunk}

I(Attention, there are some chunks which describe the file version and then is the edit chunk.) 
+--EDIT3DS (0x3D3D)//Edit chunk

| | The following are edit chunks.

| +--EDIT_MATERIAL (0xAFFF)

| +--EDIT_CONFIG1 (0x0100)

| +--EDIT_CONFIG2 (0x3E3D) //Configuration edit chunk

| +--EDIT_VIEW_P1 (0x7012)//Definition of Viewport

| +--EDIT_VIEW_P2 (0x7011) // Definition of Viewport

| +--EDIT_VIEW_P3 (0x7020) // Definition of Viewport

$$
\text { | +--EDIT_VIEW1 (0x7001)//Viewport }
$$

| +--EDIT_BACKGR (0x1200)

| +--EDIT_AMBIENT (0x2100)

| +--EDIT_OBJECT (0x4000)//Object chunk

| +--EDIT_UNKNW01 (0x1100)

| +--EDIT_UNKNW02 (0x1201)

| +--EDIT_UNKNW03 (0x1300)

| +--EDIT_UNKNW04 (0x1400)

| +--EDIT_UNKNW05 (0x1420)

| +--EDIT_UNKNW06 (0x1450)

| +--EDIT_UNKNW07 (0x1500)

| +--EDIT_UNKNW08 (0x2200)

| +--EDIT_UNKNW09 (0x2201)

| +--EDIT_UNKNW10 (0x2210)

| +--EDIT_UNKNW11 $(0 \times 2300)$

| +--EDIT_UNKNW12 (0x2302)

+--EDIT_UNKNW13 (0x2000)

+--EDIT_UNKNW14 (OxAFFF)

| +--KEYF3DS (0xB000) //Key Frame chunk.

Table 1. Internal Structure of Object

\begin{tabular}{|l|l|}
\hline ID & Description \\
\hline 4110 & Vertex List \\
\hline 4111 & VertexOptions \\
\hline 4120 & Face List \\
\hline 4130 & Face Material \\
\hline 4140 & Mapping Coordinates \\
\hline 4150 & Face smoothing group \\
\hline 4160 & Translation Matrix \\
\hline 4165 & Object visible/invisible \\
\hline 4170 & Standard Mapping \\
\hline
\end{tabular}

As shown above, the ID0x400 sub chunk is an object description chunk and it can describe objects, light sources, and cameras. The $0 \times 4000$ sub chunk contains a triangularpolygon list sub chunk $(0 \times 4100)$, a light source chunk $(0 \times 4600)$ and a camera chunk $(0 \times 4700)$. Table 1 shows the internal structure of triangular list. 


\subsection{Introduction of Maya and Ma/Mb Format}

As another Autodesk's 3D modeling software, Maya is powerful and owns a perfect system of 3D modeling and it is the most popular three-dimensional animation software currently. Compared with $3 \mathrm{ds}$ Max, Maya is more powerful in character animation and kinematics simulation. Maya is more excellent at modeling, particle systems, hair, and plants creating. In terms of modeling, Maya can create all the models created by Max and the effect is better. Maya is mainly used for film and television fields [8].

Maya has two default storage formats, respectively, in form ma and mb. Where ma represents the ASCII Maya file, mb represents the binary file. Maya is typically stored as mb format. There are more output nodes in ma and also because of the ASCII code; the ma file will be larger than the mb file when they are for the same model.

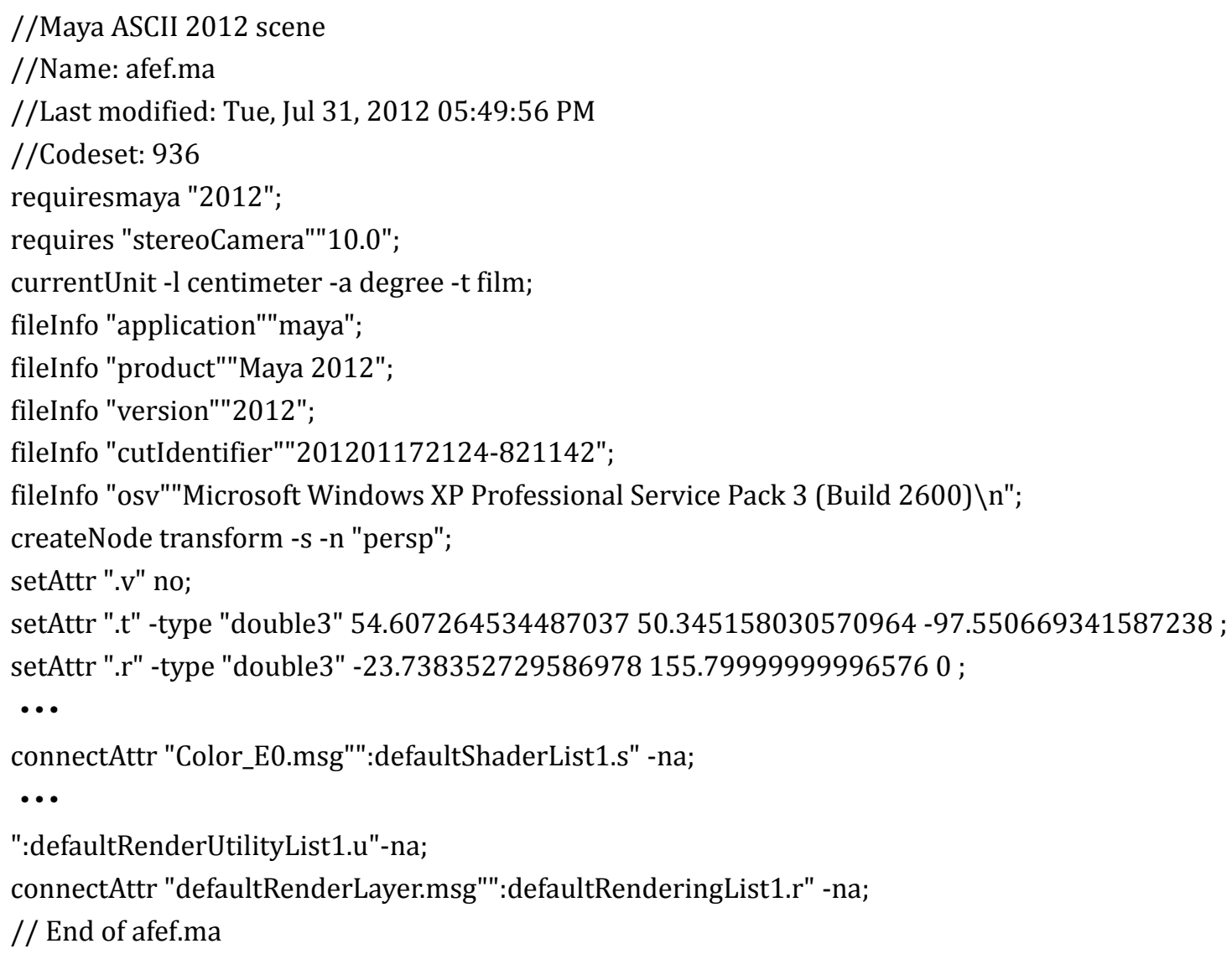

As shown above, the ma file records the version information of the model, the attributes of each node, and operations in accordance with these nodes. Along with those operations the final $3 \mathrm{~d}$ model can be recovered. mb file is binary, so it is garbled when opened in WordPad. mb file can be viewed in Ultra Edit. $\mathrm{mb}$ file also records the information of each node.

Compared to the mb file, ma file's content is more abundant, it can be opened in different versions of the Maya software, and if the file is damaged, you can open the ma file directly to modify the error.

\subsection{OpenFlight Format}

OpenFlight format is a kind of logical scene description database based on hierarchy. It consists of three parts: geometric object data, attribute and hierarchy. Geometric object data is the coordinate set. Property contains the contents of the texture and material. Hierarchical structure is to organize the data nodes in a hierarchical logical structure; it is suitable for real-time display [9].

As shown in Figure 4, OpenFlight contains the database header node, group node, object node, face node, 
vertex node, curve node, grid node, detail hierarchy node, degree of freedom node, light source and etc.

Compared to 3ds or ma files, OpenFlight format files are very different. In order to convert those files into OpenFlight format,3ds/ma file need to read the data and then store those data in OpenFlight format.

MultiGen-Paragim provides users with OpenFlight API, so that users can convert models by the giving API, do real-time simulation, do automatic modeling or expand the function of Creator in the form of plug-ins. OpenFlight API consists of four modules: Read, Write, Extensions, and Tools. Read module can read, travel and query OpenFlight database; write module can write, create and modify hierarchy nodes in OpenFlight database; extensions module can define expanded data structure to add new attributes to existed nodes, or create a custom node; tools module is used to create embedded creator plugin[10]. Format conversion needs to use the Write module.

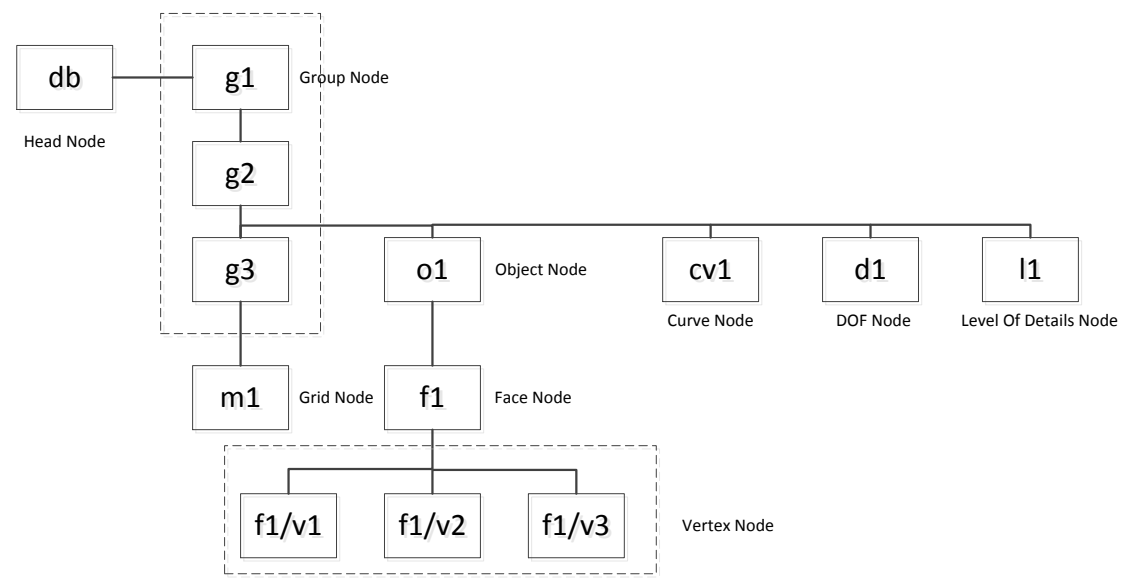

Fig. 4. Instructure of OpenFlight model.

\section{Model Format Conversion}

There are a large number of open source 3D model format conversion tools. But most tools have problems with converting to OpenFlight file. They often lose material and textures. For the mainstream modeling tools, their developers also provide a format conversion function. Take Maya as an example, its default storage format is $\mathrm{mb}$, ma, but through the Maya software we can also store the model in other formats, such as the commonly used STL, FBX, IGS and etc. Here you can convert the ma/mb file to STL or FBX file, and then use 3DS Max convert it to 3ds file. 3ds Max supports a lot of file formats, so this paper uses 3ds Max as the intermediate software. Of course, other format conversion tools can be used, such as Polytrans. Polytrans is a professional format conversion tool, it support format conversion between most of the current available formats. Polytrans also supports flt. However, as with the $3 \mathrm{~d}$ Max, converting by Polytrans will cause the problem of the loss of materials and maps. Since 3ds Max2010, 3ds Max has begun to provide the function of exporting flt file. But because of material and texture missing in the process, in this paper self-writing program is used for conversion. In Section 2.1 3ds file structure is introduced, in Section 2.3 OpenFlight file structure is introduced, two file formats can be found quite different by comparing, but they still has a great similarity in 3D data and other basic data. Therefore, the idea of file format conversion is that writing the data in 3ds file into flt file's corresponding areas.

The program flow chart is shown in Fig. 5. Reading 3DS file by using 3ds read class, then dealing with the $3 \mathrm{~d}$ s object description chunk, dealing respectively with the face chunk, node chunk, material chunk, finally using the write module in OpenFlight API to write, finally completing file format conversion [11]. For the $3 \mathrm{ds}$ object description chunk, in order to facilitate the operation in the OpenFlight, we should create a group 
node in the front of the file and then create the object description chunk relative to the Object node.

We should pay attention to dealing with the material and texture module. Because the material in $3 \mathrm{~d}$ s file chunk contains materials and textures, and in OpenFlight file textures and materials are relatively independent, so material and texture should be combined with the data in face chunk and texture mapping chunk respectively and then be written into different OpenFlight nodes.

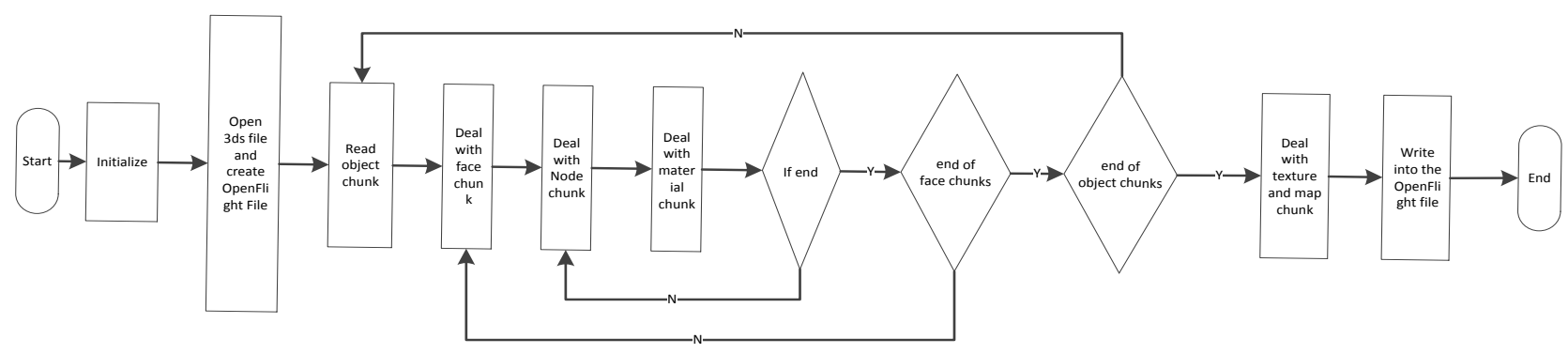

Fig. 5. Program flow.

\section{Model Optimization}

The model which is converted from other formats cannot be directly used for Prime Vega simulation. Because the 3ds Max, Maya and other modeling tools render models after creating, these non-real-time modeling tool can spend a lot of time to get a good visual effect in later time, so their model contains very rich details. That means there are a large number of faces, high resolution textures, delicate texture and rich light in the model. These details also mean a huge amount of data. If we use these models to develop the simulation application, there will be a huge challenge to the hardware, and it can hardly meet the requirements of real-time. In order to achieve the balance between visual effect and real-time performance in the simulation, the model should be simplified.

\subsection{OpenFlight Format}

Those geometric objects in the origin file have been translated into OpenFlight nodes, but due to the different habits of modelers, we still have to readjust the relationship between these nodes. According to the requirement of simulation application, some parts of the model should be separated into individual nodes in order to facilitate the setting of DOF nodes.
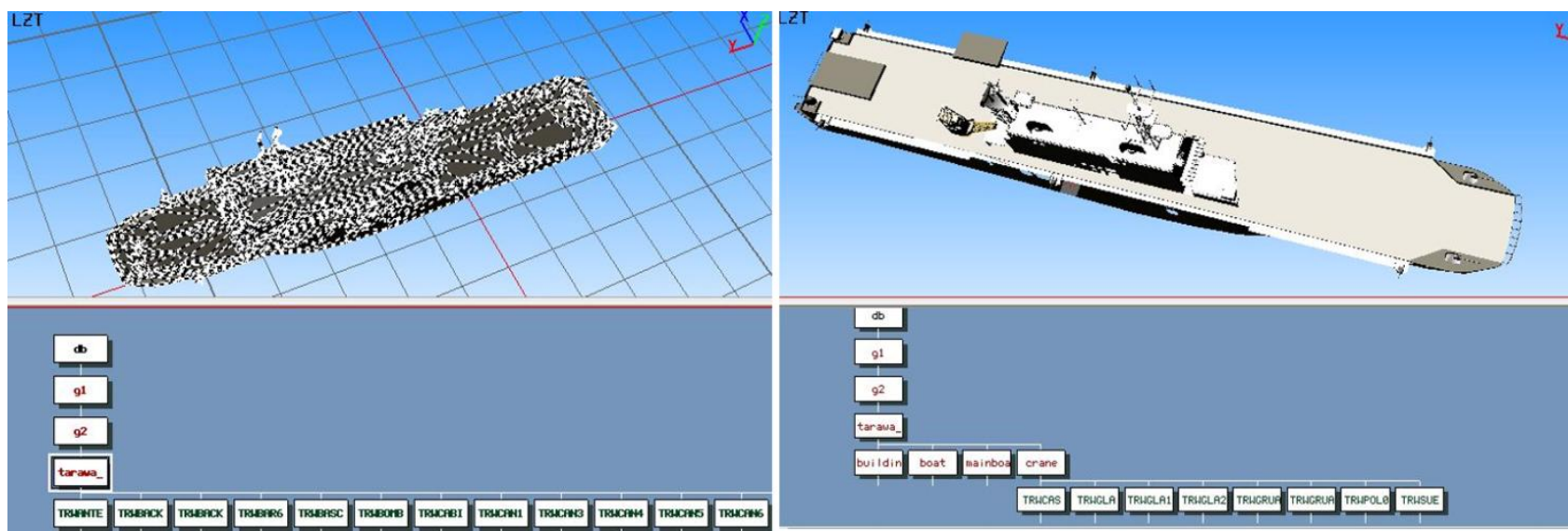

Fig. 6. Comparison of hierarchy details between adjusting and no adjusting.

Figure 6 shows the comparison of hierarchy between the file adjusted and the file not adjusted. In the left 
of picture shows the hierarchy of the file not adjusted, in this file all model geometry components are separate objects, and the model is too large, its size is still $9 \mathrm{M}$, it has nearly 4 million surfaces. When the root node is spread out, there are too much sub nodes to find a needed node to the set DOF node. Classifying these nodes is needed in order to simulation. The same parts of assembly geometry should be in a group. At the same time these parts which had no influence on the appearance of the visual effect or simulation can be removed. These operations can reduce the complexity of the model, and in the picture these operations effectively reduce the about 3000 surfaces.

After hierarchy adjustment of the model file, the hierarchy and structure is much clearer, the needed node can be quickly found.

\subsection{Structure Simplification}

Many models is a repetition of the original object, they contains a large number of internal structures. In most cases these internal structures are not needed. Those structures which would not affect the external features could be removed. We can optimize by manually and can also use VSimplify, Polytrans, Rational Reducer and other plug-ins to optimize. In this paper, we use Polytrans. We need use combine faces (merge face tool) to merge adjacent surfaces which have the public side into a single.

Figure 7 shows the comparison of faces and vertexes in Section 4.1 of the model between not simplified and simplified by VSimplify. Due to 3ds Max's requirement of visual effect, its model owns a large number of triangular patches. The existence of these triangles greatly increases the complexity. VSimplify can reduce number of patches effectively. While without the optimization, aircraft carrier model's face number is 38,275 , after optimization the number become 26,555. Face number reduces more than 10,000 , and the visual effect of the model basically has no big difference, and file size also drops from $9 \mathrm{M}$ to less than $4 \mathrm{M}$.

This step should be operated after the operations in Section 4.1. Then we can travel through these components. For each component VSimplify plug-in can be used to optimize it. For the parts which are difficult in optimizing and don't need too many details, we can manually using simple geometry to replace them. That effect is relatively good.In this step of structure simplification, Creator also provides LOD (Level Of Detail) technology which is the level of detail simplification technology. According to the different distance between the viewpoint and the model, the model can display different levels of detail. When the distance is far, it would show less details of the model. Obviously, the technology has a good gain on real-time simulation, but LOD tools provided by Creator cannot handle with complex models and its low detail model's visual effect is poor, so that tool is not recommended [12].
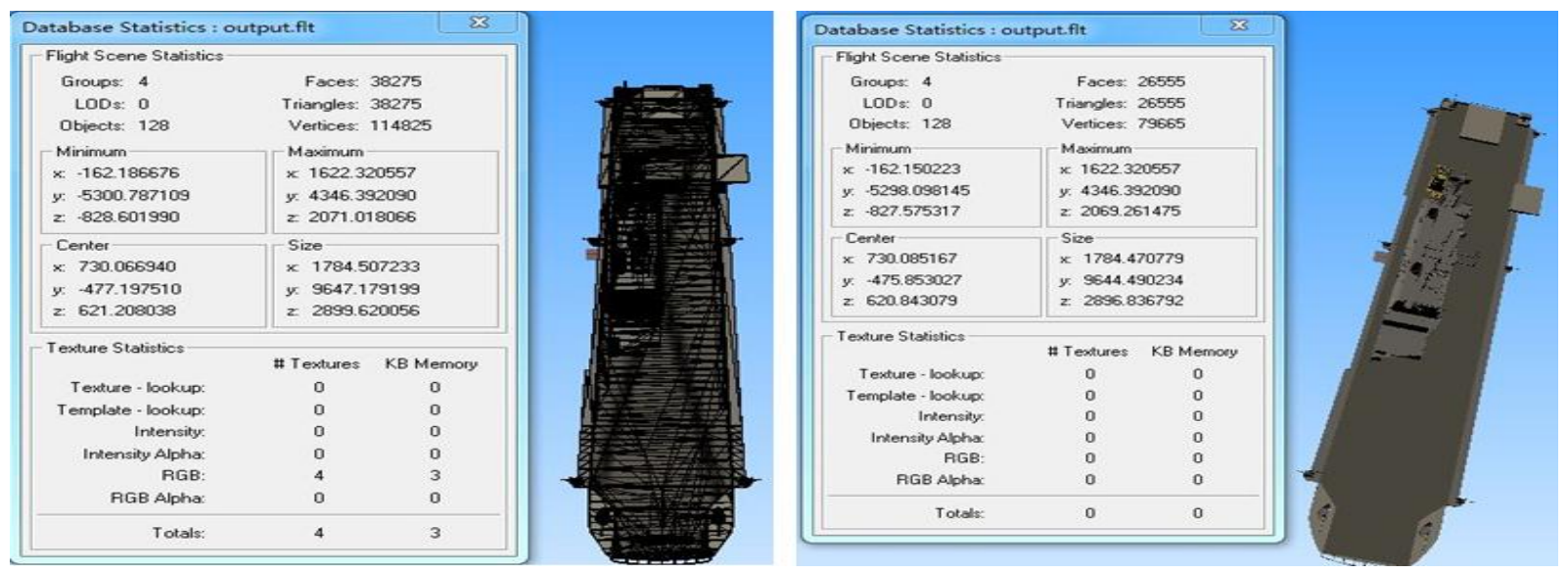

Fig. 7. Comparison of numbers of faces after optimization by vsimplify. 


\subsection{Texture Simplification}

The map is a way to simplify the model, and it can also improve the visual effect. Using texture and maps can be very effective to reduce the complexity of the model, and that way will not add geometric components. But many models use a higher resolution map in order to get realistic effects,. These high resolution textures also make the size of model file data bigger, the map could be replaced with low resolution. In the process of simulation, the texture mapping with different resolutions is not much different. In some cases, $256 \times 256$ maps can be replaced with a $16 \times 16$ map, the visual effect gap is small, but the file size is one percent of the original. For some large map, we can also reduce the texture map. As shown in Figure 8, the left model is with a resolution of $256 \times 256$ map, the map will be simplified to $128 \times 128$. It can be seen that the gap between visual effects is not big. By simplifying the map, we can further reduce the size of the model and reduce the burden of the simulation platform.
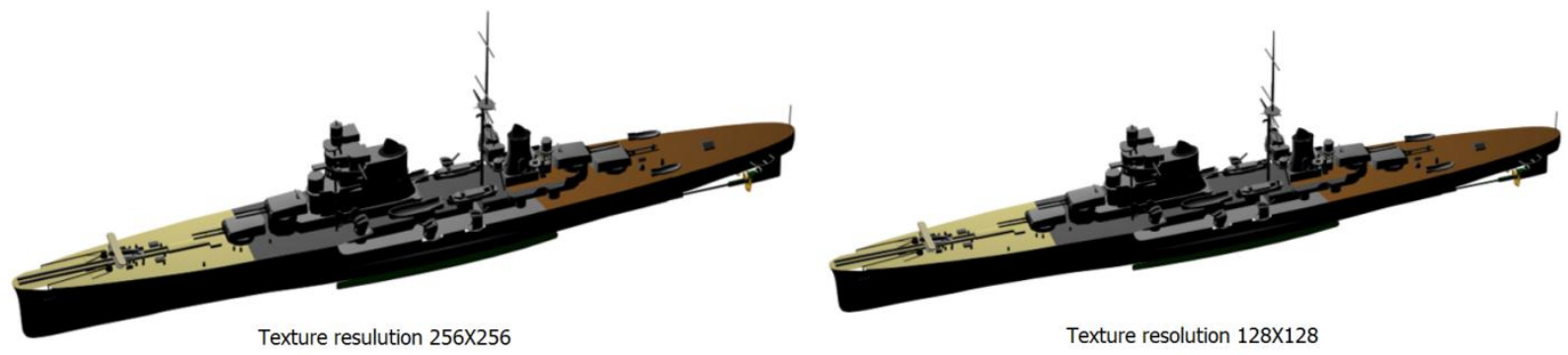

Fig. 8. Comparison of different texture resolutions.

\section{Conclusion}

In this paper, the 3D models which are built by other non-real-time rendering modeling tools are introduced into Prime Vega simulation, which greatly expand the model base. But the structure of the model created by non-real-time rendering modeling tool is too complex, that put great pressure on the simulation software and simulation platform, simulation effect will not be good, the model would make the scene cannot achieve good frames per second. In order to solve this problem, a series of model simplification methods are studied, and finally the model is simplified to the degree of visual simulation by those methods, and the balance between visual effect and real-time performance is achieved. The feasibility of introducing other modeling platform and model to simulation process has been proved, and the purpose of speeding up the process of simulation application development is successfully achieved. Those methods also provide a new way for those who lack the ability of building models.

\section{Acknowledgment}

This work is partly supported by National Natural Science Fund of China (No.61374186, No.61573131).

\section{References}

[1] Zheng, Li. P., Li, G. Y., \& Sha, J. (2007). Survey of urban simulation technology. Journal of System Simulation, 19, 2860-2863.

[2] Zheng, R., Zhang, G. F., \& Dai, S. L. (2008). Development of scene simulation platform with online edit ability. Journal of System Simulation, 20, 1229-1233.

[3] Huang Y. Y. (2014). System Simulation and Performance Evaluation Technology for Military and Civilian Areas. Beijing: National Defense Industry Press. 
[4] Yan, C. S., Huang, Y. Y., \& Wang, J. Y. (2015). Visual modeling and simulation technology of loading process for landing craft. Command Information System and Technology, 6, 73-80.

[5] Laura, B., Alessia, P., \& Francesca, F. (2015). The impact of the software's choice on dynamic daylight simulations' results: A comparison between Daysim and 3ds Max Design. Solar Energy, 122, 249-263.

[6] Liu, F., \& Liu, X. M. (2012). Research and application on the method of 3DS file read, render and control. Proceedings of the AASRI Conference on Computational Intelligence and Bioinformatics (pp. 177-182).

[7] Li, X. B., \& Yan, K. Y. (2004). Implementation of MDT model in opengl. Computer Simulation, 21, 129-131.

[8] Adam, W. (2012). Getting started in 3D with Maya: Create a project from start to finish-model, texture, rig, animate, and render in Maya. Proceedings of the AASRI Conference on Computational Intelligence and Bioinformatics (pp. 13-46).

[9] Xiao, Y., Li, G. Y., \& Wang, W. J. (2009). OpenFlight modeling approach based on 3DS. Journal of Computer Applications, 29, 302-304.

[10]Zhou, W. G. (2007). Research on the Effective Modeling of Virtual Reality Based on CAD Data. Unpublished undergraduate dissertation, Nanjing University of Aeronautics and Astronautics.

[11] Dayong, S., David, W. W., Fernando, C., \& Liu, Y. L. (2013). An ArcScene plug-in for volumetric data conversion, modeling and spatial analysis. Computers \& Geosciences, 61, 104-115.

[12] Yao, T. X. (2014). Research and Implementation on the Algorithm Of LOD Model Simplification. Unpublished undergraduate dissertation, Lanzhou Jiaotong University.

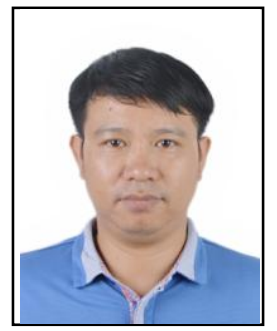

Yanyan Huang was born in Guangxi province, China in 1973. He was graduated from National University of Defense and Technology, in Changsha City, China. He received his doctor degree in systems engineering.

He is a teacher in Nanjing University of Science and Technology(NJUST) since March 2007. His research is about systems modeling \&simulation. He published in 3 books and 30 research papers. Dr. Yanyan huang is a vice Professor and doctor's tutor.

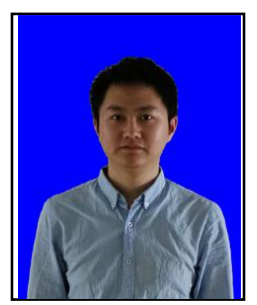

Hanghai Wu was born in Anhui province, China in 1991. He was studied for a master's degree in the field of control science and engineering in Nanjing University of Science and Technology.

He has studied in Nanjing University of Science and Technology in 2010-2014 and got a bachelor of electrical engineering. 